\title{
A Generic Danish Distribution Grid Model for Smart Grid Technology Testing
}

Cha, Seung-Tae; Wu, Qiuwei; Østergaard, Jacob

Published in:

Proceedings of IEEE PES Innovative Smart Grid Technologies (ISGT)

Publication date:

2012

Document Version

Early version, also known as pre-print

Link back to DTU Orbit

Citation (APA):

Cha, S-T., Wu, Q., \& Østergaard, J. (2012). A Generic Danish Distribution Grid Model for Smart Grid Technology Testing. In Proceedings of IEEE PES Innovative Smart Grid Technologies (ISGT) IEEE.

\section{General rights}

Copyright and moral rights for the publications made accessible in the public portal are retained by the authors and/or other copyright owners and it is a condition of accessing publications that users recognise and abide by the legal requirements associated with these rights.

- Users may download and print one copy of any publication from the public portal for the purpose of private study or research.

- You may not further distribute the material or use it for any profit-making activity or commercial gain

- You may freely distribute the URL identifying the publication in the public portal

If you believe that this document breaches copyright please contact us providing details, and we will remove access to the work immediately and investigate your claim 


\title{
A Generic Danish Distribution Grid Model for Smart Grid Technology Testing
}

\author{
Seung Tae Cha, Member, IEEE, Qiuwei Wu, Member, IEEE and Jacob Østergaard, Senior, IEEE
}

\begin{abstract}
This paper describes the development of a generic Danish distribution grid model for smart grid technology testing based on the Bornholm power system. The proposed approach uses a coherency based dynamic equivalent technique along with relation \& participation factors, and is suitable for use with real time digital simulator (RTDS). In particular, the frequency dependent network equivalent (FDNE) method has been combined with parameter randomization in order to accurately preserve the desired properties and characteristics of the original Bornholm power system. The validity and efficiency of the proposed approach is demonstrated by comparing the transient response of the original Bornholm power system model and the developed generic model under significant fault conditions. The results clearly show that the equivalent generic distribution grid model retains the dynamic characteristics of the original system, and can be used as a generic Smart Grid benchmark model for testing purposes.
\end{abstract}

Index Terms-- Generic Danish distribution grid, dynamic equivalent, frequency dependent network equivalent (FDNE), parameters randomization, real time digital simulator (RTDS), smart grid

\section{INTRODUCTION}

A real-time digital simulator (RTDS) has been in operation at Centre for Electric Technology (CET), Technical University of Denmark (DTU) as a part of the PowerLabDK experimental platform which is shown in Fig. 1. The PowerLabDK is an internationally unique experimental platform for sustainable electric power and energy systems. It is now one of Europe's most advanced research site for development and demonstration with distributed control architectures for large-scale power systems, coupled with a state-of-the-art control room with a full scale SCADA system. A fully integrated SCADA system is essential for real-time operation and control of the simulated power systems for a comprehensive laboratory environment. The platform can be used to investigate all aspects of the challenges and requirements of future power systems related to operation and control. The entire system also allows for hardware in the closed-loop (HIL) simulations including information and communication technology (ICT) infrastructure in order to investigate the future power system behaviors. PowerLabDK can be used for different types of research ranging from basic

This work was supported in part by the Transmission System Operator (TSO), Energinet DK, EnergyLab DK, and Technical University of Denmark, under the project grant 55622.

Seung Tae Cha, Qiuwei Wu and Jacob Østergaard are with Center for Electric Technology, Technical University of Denmark, DK 2800, Denmark (e-mail: stc@elektro.dtu.dk, qw@elektro.dtu.dk, joe@elektro.dtu.dk). research to large-scale, and full-scale experiments with the Bornholm power system which has more than 28,000 consumers and more than 33\% wind power penetration.

With respect to the above mentioned facility and the corresponding developments, it is very interesting and important to develop an equivalent generic distribution grid model which can replicate the performance of the Bornholm power system under both steady state and dynamic conditions. The developed generic distribution grid model will serve as a benchmark model for the research community and help other researchers replicate the test results that have been obtained using the PowerLabDK platform. In order to develop such a generic model, there are a few options, i.e. the dynamic reduction method, or a useful randomization technique for various types of parameters or something equivalent into the 'modelled' or 'simulated' power systems, or the combination.

The need of real time simulation platform stems from the above fact that in many practical situations, it is of great importance and desirable to model the power system dynamics accurately. The influence of the dynamic reduction on power system security and stability will become decisive, if the size of grid increases. In an RTDS platform, the size or number of the RTDS hardware and subsequently its monetary cost is proportional to the size of the modelled system. Hence, it makes an economic sense to model in full detail only that part of network which is of great interest, and formulate the remainder of the network into an appropriately accurate equivalent. Further, it has become beneficial to construct dynamic equivalent systems since such studies for large power system require formidable computational effort [1][2][3][4]. Also, the confidential requirements from grid companies and generation plants prevent the academia and researchers from working on a real grid model. An equivalent generic model is of great interest that does not contain the confidential information meanwhile conserves the original system characteristics or retains most properties of the original system. It is also becoming increasingly important to be able to build equivalent systems or generalized systems, which could provide similar dynamic responses as the actual grid, however avoids the detailed information on the original power system components such as generators, transformers, and lines [5][6]. Thus, a strong stimulus exists for the development of equivalent or generalized model of the Bornholm power system in order to optimize the resources necessary to analyze in detail the dynamic behaviour of the Bornholm power system. 


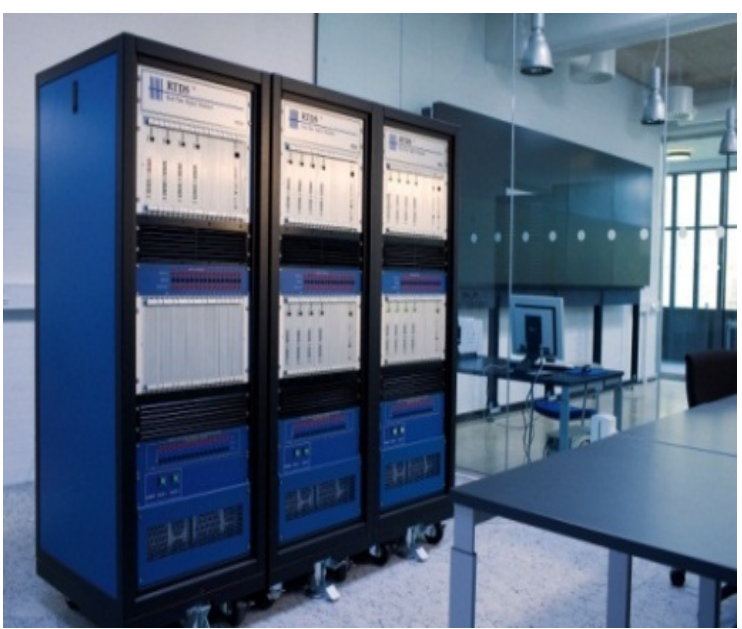

Fig. 1. Real time simulation platform at PowerLabDK

Previous work on dynamic equivalents have been reported in many literatures to improve the dynamic reduction algorithm [7][8][9][10]. It has been found that with the application of coherency approach technique, the system can be significantly reduced and improved. The generators are identified to the coherent groups according to the relation factors, with considering the measure of the relative participation of the generators in the group. All generators and controllers in the group are weighted by participation numbers and aggregated to construct a dynamic equivalent [2][3][4].

Likewise, in [1][10], an improved wide-band multi-port equivalent is presented, which reduces a large power network into a small manageable equivalent model that preserves wideband behaviours.

The proposed work builds on this dynamic equivalent approach by using the relation factors and errors between generators in order to identify the groups and aggregate the generators in the same group [4], while the parameter randomization is done using MatLab's normrnd function. Then, the randomized parameters of components are feed into the reduced RTDS model for further process which later combines with FDNE method into a simpler equivalent.

The rest of the paper is organized as follows. A brief description of the coherency, FDNE, and parameters randomization is provided in Section II. The Bornholm power system is described in Section III. The simulation results are presented in Section IV and a conclusion is drawn in Section V.

\section{PROPOSED EQuivalent}

The algorithm used in this work is based on the previous author's research in [4], and is an extension of the earlier research of [5] improving dynamic reduction of power systems using relation factors combining with FDNE feature available in RTDS. The proposed equivalent procedure is illustrated in Fig. 2. In the earlier work [4], a dynamic equivalent approach using the relation factors and errors between generators has been developed in order to identify the groups. Also, the participation factors are used to aggregate the generators in the same group. Once the coherency identification procedure creates a reduced network in which coherent groups of generators are each replaced by a single representative generator in the first step. In the second step, the network is then modified and the parameter randomization takes place in which Matlab's normrnd function is used to generate random numbers from the normal distribution with mean parameter $\mu$ and standard deviation parameter $\sigma . \mu$ is set as the original value of the distribution lines, cables, and transformers' leakage reactances, and $\sigma$ varies in the different cases. As the last step, FDNE is adopted with the above two steps to accurately capture the high frequency behaviour. Together, these three steps create an equivalent system that is valid for the Electromagnetic Transient (EMT) simulation. The advances of the FDNE algorithm are adopted from the previous research work [1][10]. The detailed procedure is available in literature [1][3][4][10][11] and hence is briefly discussed below.

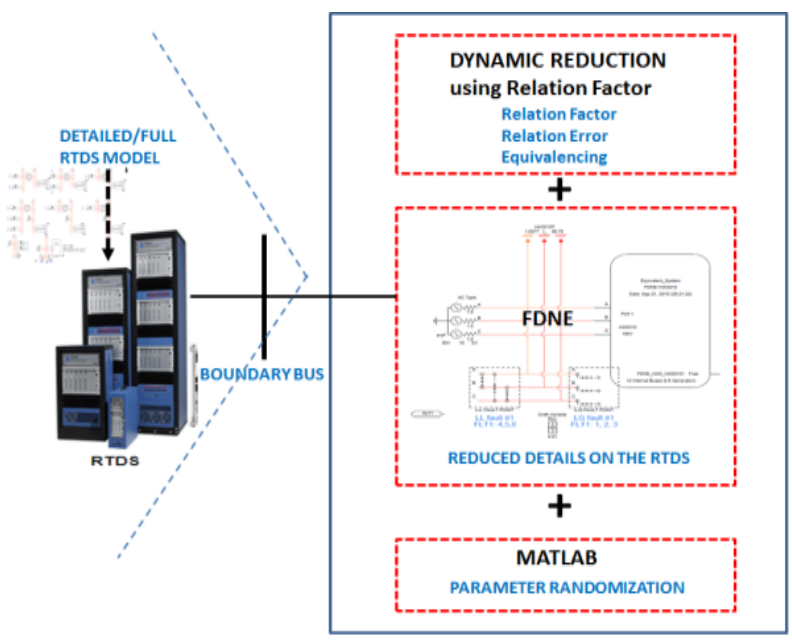

Fig. 2. Proposed equivalent procedure

\section{A. Dynamic Reduction}

A reliable coherency measure is the rotor angle and machine speed deviation. The coherency procedure is applied to the generator buses in order to reduce their numbers and is made up of three steps. In [4], the coherent group is directly identified from the state matrix according to the relation factors, which represent the relative coupling degree between the generators. The first step is the identification of groups with coherent generators, which have similar characteristics for certain disturbance. The second step is the aggregation of equivalent generators among discriminated groups. All generators and associated controllers in the coherent group are weighted by participation numbers, and aggregated to construct a dynamic equivalent. The third step is the network reduction considering the power flows and bus voltages of the original system. This coherency based network reduction procedure gives a reduced model of the network representing its electromechanical behavior. The external systems (is referred to as equivalents) or reduced systems, shown in Fig. 3 , consist of some buses such as boundary buses, retained buses and deleted buses when the network reduction is performed. 


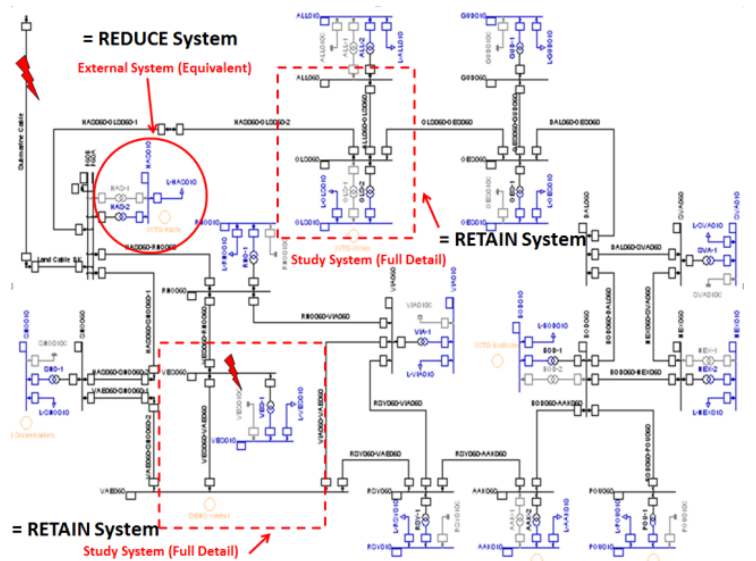

Fig. 3. Reduced system and retained systems

\section{B. FDNE}

The concept of FDNE is making a model which can be simulated in the time domain and at higher frequencies has essentially the same frequency response as the reduced network and hence has the same essential time domain response. The FDNE is a two part equivalent that provides a way of simulating larger power systems with reduced details on the RTDS. Therefore, the equivalent uses far less hardware resources (less computational processing power) compared with simulating the original system with full details and allows users to simulate a much larger overall power system using available hardware with minimum loss of accuracy [11]. The general idea of such equivalent is illustrated in Fig. 4.

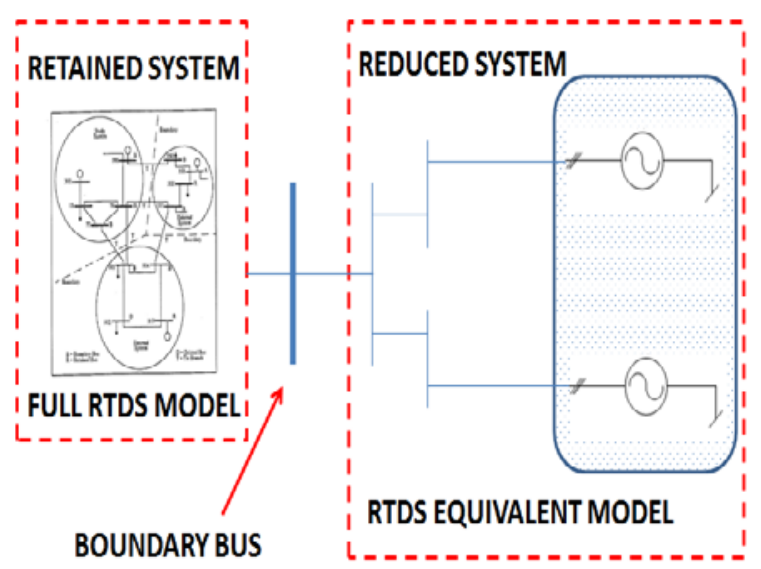

Fig. 4. General concept of FDNE

\section{Parameter Randomization}

This part of the work develops the generic model of the network. It includes two steps. In the first step, a generic topology of the network is proposed to represent the common topology patterns of the real network [5]. In parallel, the transmission/distribution lines, cables, and transformers are categorized based on their types, material properties, parameters (e.g length, leakage reactance), etc, and the generic parameter sets are developed from each category and used in the equivalent RTDS model for further process which later combines with above FDNE process into form a simpler equivalent. The line parameters of the proposed generic distribution grids are identified, averaged and randomized based on the complete data on the $60 \mathrm{kV}$ and $10 \mathrm{kV}$ lines using Matlab’s normrnd function. This is a useful technique for obtaining the randomized numbers. It generates random numbers from the normal distribution with mean parameter $\mu$, and standard deviation parameter $\sigma$. The following Fig. 5 is a simple explanation of this technique.

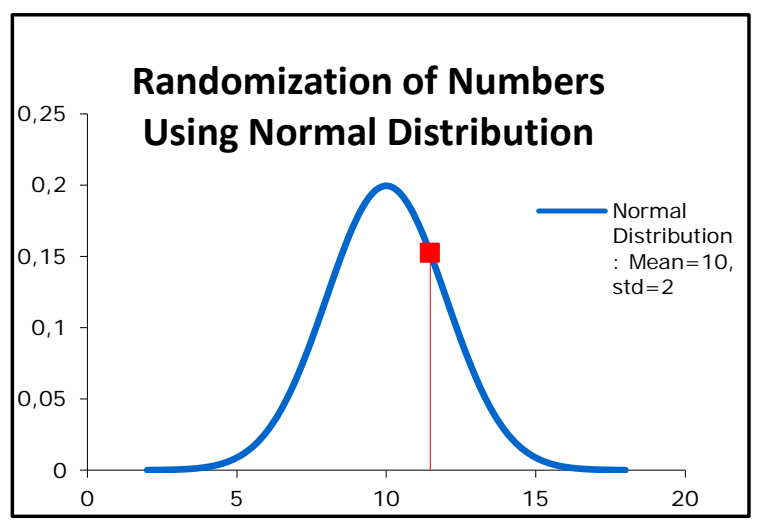

Fig. 5. Result of randomization for type $\mathrm{C}, 60 \mathrm{kV}$ distribution line

This resulted in 38 groups in which 14 of the typical categories are used in the proposed generic models, which is summarized in table I.

TABLE I

Categories of Distribution Line Types, Sizes and Properties

\begin{tabular}{|c|c|}
\hline Category & Material Properties \\
\hline Tyре A & Cab_3x1x150 PEX Al+25Cu.TypLne \\
\hline Tyре B & Ohl_3x1x130 StAl+50Fe.TypLne \\
\hline Tyре C & Ohl_3x1x130 StAl+50Fe.TypLne \\
\hline Type D & Cab_3x1x095 PEX Al+25Cu \\
\hline Type E & Cab_1x3x240 PEX Al+35Cu \\
\hline Type F & Cab_1x3x095 APBF Cu \\
\hline Tyре $G$ & Cab_3x1x300 PEX Al+35Cu \\
\hline Cable A & Island to seacable bus \\
\hline Cable B & Seacable \\
\hline Cable $C$ & Inland bus to seacable bus \\
\hline Tyре $H$ & AL095 \\
\hline Type I & AL050 \\
\hline Type J & CU050 \\
\hline Type K & CU035 \\
\hline
\end{tabular}

\section{BORNHOLM POWER SYSTEM}

The Bornholm power system is connected through a long submarine cable to the Swedish power system, represented in the model as a simple external grid. The power system contains 3 voltage levels, $60 \mathrm{kV}, 10 \mathrm{kV}$, and $0.4 \mathrm{kV}$. At $60 \mathrm{kV}$ level, the distribution network has 18 nodes, $2360 / 10 \mathrm{kV}$ transformers with on load tap changer, 22 cables and 26 overhead lines. Bornholm has a peak demand for electricity of approximately 55MW and a base load of 25MW. The 
generators include 14 diesel (oil) units with a total capacity of 35MW, 1 steam power plant with 25MW capacity, 1 combined heat and power plant with 37MW capacity, 35 wind turbines with a total capacity of 30MW, and one 2MW biogas plant. In the equivalent model, both $60 \mathrm{kV}$ and $10 \mathrm{kV}$ grids are replica of the actual grid on Bornholm. A detailed description and the parameters can be found in [12]. The Bornholm equivalent model has been implemented and the final test system used in this simulation is depicted in Fig. 6. The real system is firstly modeled entirely on the RTDS and named as 'RTDS Full Model Original' in the simulation results. As they are represented in full detail, their results can be regarded as benchmarks for validating the proposed 'RTDS Equivalent'.

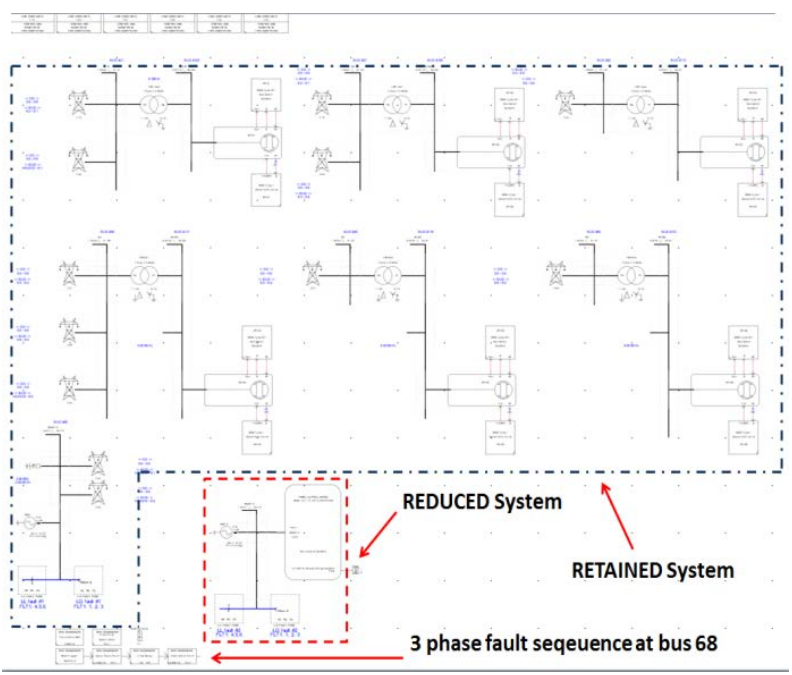

Fig. 6. RTDS equivalent generic distribution model

\section{Simulation RESUltS}

In this section, several simulations were performed in order to validate the proposed equivalent approach. A three phase fault of 6 cycle duration on bus 68 is simulated. The simulation results of the RTDS Full Model Original and RTDS Equivalent Model are shown in Fig. 7. It can be seen from the Fig. 7 (a) - 7(b) that the active and reactive power of generator \#1. The first one is shown with legend RTDS Full Model Original (blue curves) and the second one is the proposed RTDS Equivalent (green curves), respectively.

Fig. 7(c) shows the transient behaviour of the relative generator speed after the disturbance.

Fig. 8 shows the three phase voltage of the bus 68 of the retained and reduced system. Two curves are shown. The first one is shown with legend RTDS Full Model Original (blue curves) and the second one is the proposed RTDS Equivalent (green curves), respectively. The two curves are nearly identical and appear as one, strongly indicating that the proposed method provides an accurate equivalent of the external system.

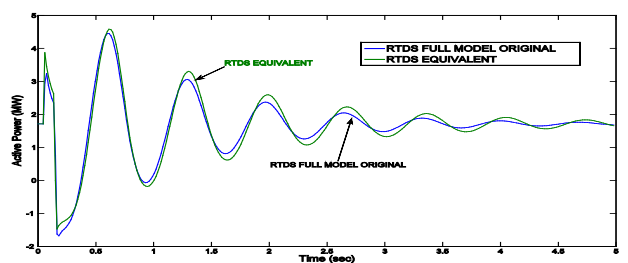

(a)

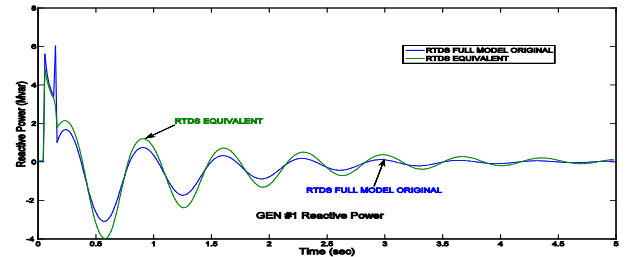

(b)

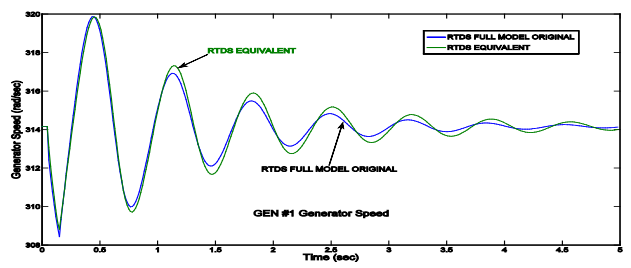

(c)

Fig. 7. Comparison of RTDS Full Model and RTDS Equivalent is subjected to a three phase fault of 6 cycle duration on bus 68 (a) generator $\# 1 P_{\text {out }}$, (b) generator \#1 $Q_{\text {out }}$, (c) generator \#1 rotational speed

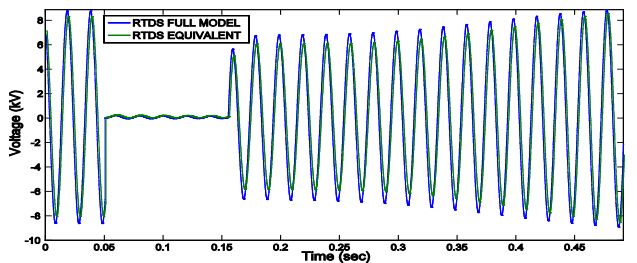

(a)

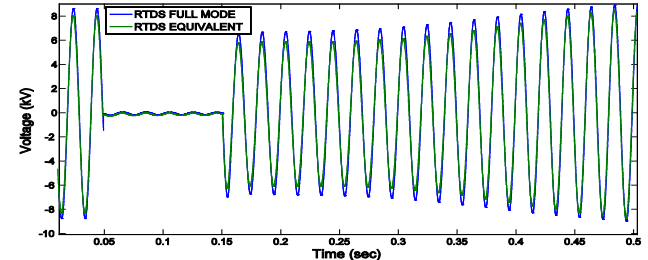

(b)

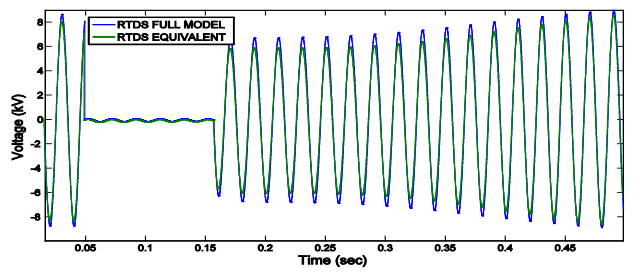

(c)

Fig. 8. Comparison of RTDS Full Model and RTDS Equivalent is subjected to a three phase fault of 6 cycle duration on bus 68 (a) A phase voltage, (b) B phase voltage, (c) C phase voltage 
The result of the RTDS equivalent is very close to the benchmark of RTDS Full Model Original. As the captured plots agree very closely, the RTDS equivalent of the external system can reproduce the dynamic response of the full external system.

As an additional comparison, a sudden load change is presented during islanding operation mode. The load was varied from $3 \mathrm{MW}$ to $4 \mathrm{MW}$, while the simulation is running, in order to simulate an increase in load demand. The main results obtained are as shown in Fig. 9. It can be seen from the Fig. 9(a)-(b) that both the active power of generator unit 5 and 6 increase from 5.25 MW to 5.6 MW, and $13 \mathrm{MW}$ to $13.6 \mathrm{MW}$ to control the overall island frequency and voltage by sharing or rescheduling between two units based on their participation factors. Fig. 9(c) show the frequency deviation caused by the wind variation.

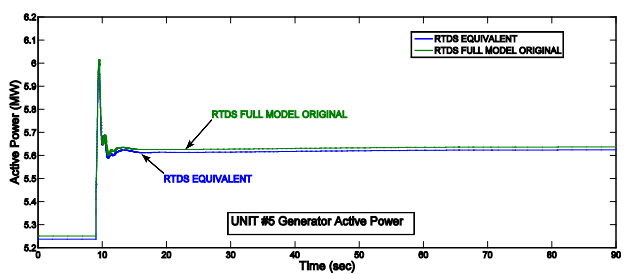

(a)

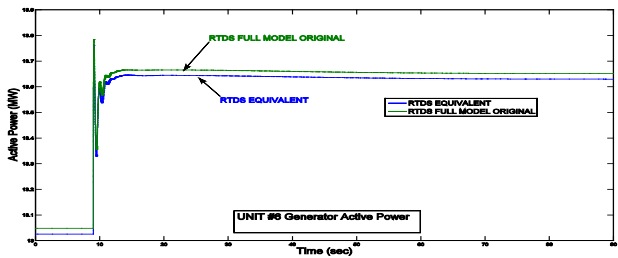

(b)

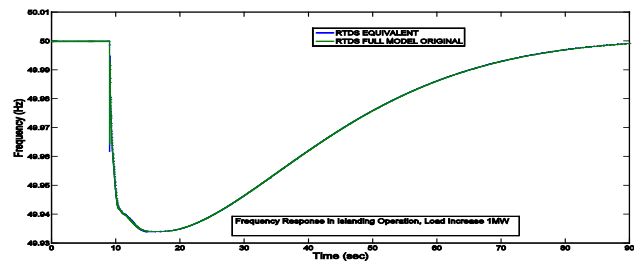

(c)

Fig. 9. Comparison of RTDS Full Model and RTDS Equivalent is subjected to $1 \mathrm{MW}$ load increase at $60 \mathrm{kV}$ during islanding operation mode (a) generator \#5 $P_{\text {out }}$, (b) generator \#6 $P_{\text {out }}$, (c) system frequency

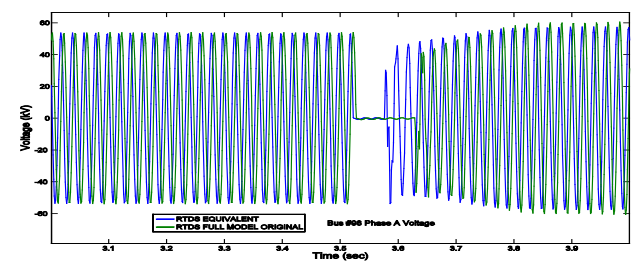

(a)

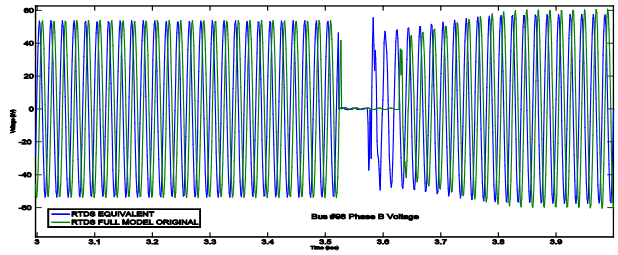

(b)

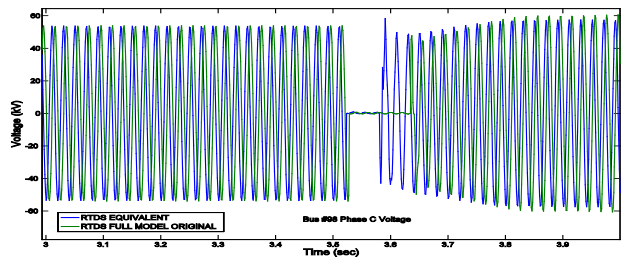

(c)

Fig. 10. Comparison of RTDS Full Model and RTDS Equivalent is subjected to a three phase fault of 6 cycles duration on bus 98 during islanding operation mode (a) A phase voltage, (b) B phase voltage, (c) C phase voltage

Fig. 10 shows the three phase voltage of the bus 98 of the retained and reduced system. Two curves are shown. The first one is shown with legend RTDS Full Model Original (blue curves) and the second one is the proposed RTDS Equivalent (green curves), respectively. The two curves are nearly identical and appear as one, strongly indicating that the proposed method provides an accurate equivalent of the external system and retains most property of the original system.

The results obtained in Fig. 10 demonstrate very good agreement between the RTDS Full Model Original and RTDS equivalent model.

\section{CONCLUSION}

This paper proposed a procedure to constitute an equivalent generic system of a real power system whose scale is reduced within the hardware capacity for the real time digital simulator, and the results exhibit good agreement between the RTDS full model original and the RTDS equivalent. Two resultant curves match to a very high degree of accuracy, which gives the confidence that the equivalent approach and network equivalents have been performed properly.

\section{ACKNOWLEDGEMENT}

The work was supported by the Transmission System Operator (TSO), Energinet.dk, the Distribution System Operator (DSO), Østkraft, Bornholm and Technical University of Denmark (DTU). The authors gratefully acknowledge the support received from Dr. Ming and Dr. Liang, RTDS Technology and Christian B. at DTU.

\section{REFERENCES}

[1] Xi Lin, A. M. Gole, and Ming Yu, "A wide-band multi-port system equivalent for real-time digital power system simulators," IEEE Transactions on Power Systems, vol. 24, No. 1, pp. 237-249, 2009.

[2] Gilsoo Jang, H. J Kim and Y.B Yoon, "Development of equivalent systems for a KEPCO enhanced power system simulator," IEEE Power Engineering Society Winter Meeting, vol. 3, pp. 1685-1688, 2000.

[3] H. J. Kim, G. Jang, and K. Song, "Dynamic reduction of the large-scale power systems using relation factor," IEEE Transactions on Power Systems, vol. 19, No. 3, pp. 1696-1699, 2004.

[4] Yong Hak Kim, Seung Tae, Cha, J.W Lee, T.K. Kim, J. B. Choo, and H.K Nam, "Construction of largest equivalent systems for power system 
simulator," pp. 79-91, European Transactions on Electrical Power, 2005.

[5] Rasmus H. Rasmussen, Theis N. Nielsen, Guang-Ya Yang, G. Rodrigo G. Valle, and Jacob Østergaard, "Dynamic equivalent model of a Danish island for MV and LV studies," IEEE ISGT Europe, pp. 1-4, 2011.

[6] M. Artenstein, A. Giusto, "Equivalent model of the Argentinian electrical power system for stability analysis of the Uruguayan network," IEEE Transmission \& Distribution Conference and Exposition, pp. 1-5, 2008.

[7] J. Zaborsky, K. W. Whang, "A clustered dynamical model for a class of linear autonomous systems using simple enumerative sorting," IEEE Transactions on Circuit and Systems, vol. 29, No. 11, pp. 747-757, 1982.

[8] A. J. Germond and R. Podmore, "Dynamic aggregation of generating unit models,” IEEE Transactions on Power Systems, vol. 97, pp. 10601069, 1978.

[9] R. Podmore, "Identification of coherent generators for dynamic equivalents," IEEE Transactions on Power Systems, vol. 97, pp. 13441354, 1978.

[10] Yuefeng Liang, Xi Lin, A. M. Gole, Ming Yu, "Improved coherencybased wide-band equivalents for real-time digital simulators," IEEE Transactions on Power Systems, vol. 26, No. 3, pp. 1410-1417, 2011.

[11] RTDS FDNE An Introduction Manual, 1-18

[12] Seung Tae Cha, "RTDS implementation of Bornholm power system distribution system modeling and analysis," Dept. Electrical Eng., Technical University of Denmark, Lyngby, Internal Report, pp. 1-58, Nov. 2010.

\section{BIOGRAPHIES}

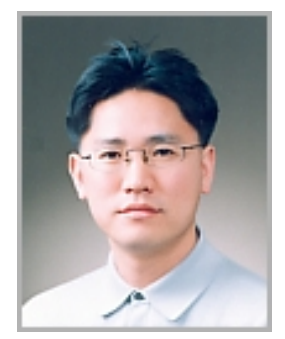

Seung Tae, Cha received his B.S degree in Electrical Engineering from Illinois Institute of Technology, Chicago, U.S. in 1992 and M.S degree in Electrical Engineering from Yonsei University, Korea in 1997. Upon graduation, he joined Korea Electric Power Research Institute, KEPCO where he was actively engaged in the development of KEPCO's Enhanced Power system Simulator (KEPS), a fully digital real-time simulator, and other various power system related research projects. He is a Ph.D candidate at Technical University of Denmark \& Korea University. His research interests include real-time simulation of power systems, power hardware-in-the-loop (PHIL) testing, integration of renewable energy resources, model development, agent-based simulation \& optimization, studies involving load flow, system planning and operation.

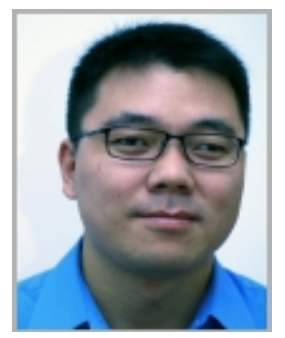

Qiuwei Wu (M' 09) obtained the B. Eng. and M. Eng. from Nanjing University of Science and Technology, Nanjing, P. R. China, in 2000 and 2003, respectively, both in Power System and Automation. $\mathrm{He}$ obtained the $\mathrm{PhD}$ degree from Nanyang Technological University, Singapore in 2009 in Power System Engineering.

He worked as a senior R\&D engineer in Vestas Technology R\&D Singapore Pte. Ltd. from Mar. 2008 to Oct. 2009. He joined Centre for Electric Technology (CET), Department of Electrical Engineering, Technical University of Denmark (DTU) as PostDoc in Nov. 2009 and has been an assistant professor with CET since Nov. 2010.

His research interests are integration of electrical vehicles (EVs) into power systems for high penetration of renewable energy sources (RES), integration study for wind power, dynamic performance of power systems with high penetration of RES, real time simulation of power systems using RTDS and reliability analysis and improvement of restructured power systems using demand response programs.

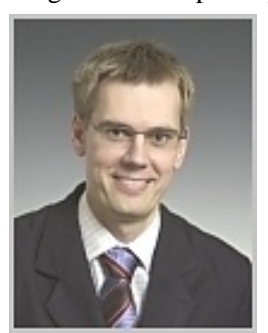

Jacob Østergaard (M'95-SM' 09) obtained his MSc in Electrical Engineering from Technical University of Denmark (DTU) in 1995.

He was with Research Institute of Danish Electric Utilities for 10 years where he did research within power system transmission and distribution and was responsible for developing industrialacademic collaboration. Since 2005 he has been
Professor and Head of Centre for Electric Technology (CET), DTU. His research interests cover SmartGrids with focus on system integration of renewable energy and distributed energy resources, control architecture for future power system, and flexible demand.

Prof. Jacob is serving in several professional organizations, boards and steering committees. He is head of the Danish experimental platform for electric power and energy, PowerLabDK, and he has been member of the EU SmartGrids advisory council. In 2009 he received the IBM Faculty Award. 